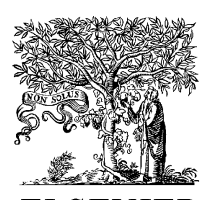

ELSEVIER

\title{
Preparation and characterization of MCM-41 and silica supported nickel boride catalysts
}

\author{
She-Tin Wong ${ }^{\mathrm{a}}$, Jyh-Fu Lee ${ }^{\mathrm{b}}$, Jin-Ming Chen ${ }^{\mathrm{b}}$, Chung-Yuan Mou ${ }^{\mathrm{a}, *}$ \\ a Department of Chemistry, National Taiwan University, \#1, Section 4, Roosevelt Road, Taipei, Taiwan 106, ROC \\ b Synchrotron Radiation Research Center, Hsinchu, Taiwan, ROC
}

Received 24 April 2000; received in revised form 27 July 2000; accepted 27 July 2000

\begin{abstract}
$\mathrm{Ni}-\mathrm{B} / \mathrm{MCM}-41$ and $\mathrm{Ni}-\mathrm{B} / \mathrm{SiO}_{2}$ catalysts were prepared by low-temperature reduction of surface-adsorbed nickel chloride with ethanolic sodium borohydride solution. XRD analysis showed that the MCM-41 framework of Ni-B/MCM-41 catalyst remained intact, while nitrogen adsorption-desorption study revealed uniform pore size distribution of the channel system. Thermal treatment of this catalyst under nitrogen at $450^{\circ} \mathrm{C}$ indicated the presence of a segregated nickel phase on the surface of MCM-41. On amorphous silica, however, amorphous nickel boride alloy may have formed. As-synthesized Ni-B/MCM-41 is more susceptible to oxidation than $\mathrm{Ni}-\mathrm{B} / \mathrm{SiO}_{2}$ probably due to the smaller size of its alloy particles. This suggestion is also supported by the EXAFS results. The lower activity of $\mathrm{Ni}-\mathrm{B} / \mathrm{MCM}-41$ catalyst than $\mathrm{Ni}-\mathrm{B} / \mathrm{SiO}_{2}$ in benzene hydrogenation reaction probably reflects the lower reducibility of nickel oxide phase than nickel boride alloy phase. (C) 2001 Elsevier Science B.V. All rights reserved.
\end{abstract}

Keywords: Sodium borohydride; Nickel boride; MCM-41; Amorphous alloy; Benzene hydrogenation

\section{Introduction}

Amorphous alloys have attracted much attention in the past two decades due to their special physical and chemical properties [1,2]. In particular, they have found important practical or potential applications in various fields, such as in powder metallurgy, composite materials, and catalysis. With the unique combination of small particle size and short-range order and long-range disorder structure, these ultrafine amorphous alloys have gained increasing attention in catalysis. Ever since the first report on catalysis with amorphous alloy catalysts by Smith et al. [3], a lot of works on this subject have been published. However,

\footnotetext{
${ }^{*}$ Corresponding author. Fax: +886-2-23660954.

E-mail address: cymou@ms.cc.ntu.edu.tw (C.-Y. Mou).
}

wide application of these catalysts is restricted due to their low thermal stability or crystallization temperature.

Recently, there is a renewed interest on the study of metal boride (M-B) or metal phosphide (M-P) alloy catalysts. Now, supported alloy catalysts with better thermal stability were prepared by the chemical reduction method. This method has many advantages over the conventional melt-quenching method used for preparing amorphous alloy ribbons [4]. For example, amorphous silica with low surface area $\left(\approx 200 \mathrm{~m}^{2} / \mathrm{g}\right)$ has been widely used as the support and a range of new hydrogenation catalysts has been reported. These new alloy catalysts are more active and selective than the corresponding metal catalysts $[5,6]$. Some interesting aspects covered by these studies include the sulfur-resistance of nickel boride catalyst 
and its high catalytic stability towards highly reactive reactants such as dienes. Naturally, if a support of higher surface area is use, then better dispersed, and hence, more stable catalyst can be prepared. MCM-41 with high surface area and porosity could be a better catalyst support than amorphous silica [7]. Most importantly, its mesoporous channels allow diffusion of bulky unsaturated compounds. These unique features of MCM-41 motivate us to study the MCM-41 supported nickel boride catalysts, denoted hereafter as Ni-B/MCM-41.

In this study, we shall describe our preliminary results on the preparation and characterization of Ni-B/MCM-41 catalyst. The true nature of the surface species on MCM-41 is of particular interest to us, i.e. whether they have the same type of amorphous alloy nature as reported for $\mathrm{Ni}-\mathrm{B} / \mathrm{SiO}_{2}$. Our discussion focuses on three important aspects: (1) the influence of catalyst synthesis conditions on the stability of MCM-41 structure, and (2) the influence of MCM-41 channel structure on the composition of the surface species, and (3) the influence of oxygen on the stability of the surface species. The latter is important since passivation of the catalyst by exposing to air made sample handling more convenient. Passivation of ruthenium metal surface has been described for $\mathrm{Ru} / \mathrm{SiO}_{2}$ catalyst in catalytic hydrogenation of benzene [8].

\section{Experimental}

\subsection{Preparation of $\mathrm{Ni}-\mathrm{B} / \mathrm{MCM}-41$ and $\mathrm{Ni}-\mathrm{B} / \mathrm{SiO}_{2}$ catalysts}

Pure silica MCM-41 was synthesized according to the well-established method of [9] with hexadecyltrimethylammonium bromide (CTAB) as the templating agent. Amorphous silica was commercially available (Acros) with a surface area of about $500 \mathrm{~m}^{2} / \mathrm{g}$.

The preparation of supported Ni-B catalysts consists of two stages. About $0.6 \mathrm{~g}$ of MCM-41 or $2.0 \mathrm{~g}$ of silica was first impregnated with nickel chloride hexahydrate (10-30 wt.\% of nickel) in methanol via an incipient wetness technique. After removing the solvent at $50-60^{\circ} \mathrm{C}$, the solid was left at $100^{\circ} \mathrm{C}$ for about 2 days to give a golden-yellow powder. The reduction process was then initiated by adding a saturated solution of sodium borohydride in $99.5 \%$ ethanol $(\mathrm{B} / \mathrm{Ni}$ molar ratio $=0.7-6$ ) directly to the powder maintained at ice-bath temperature and stirred vigorously. In some cases, a little excess of ethanol was added in order to allow the stirring to proceed smoothly. The reduction process lasted for $0.5 \mathrm{~h}$ until there was no gas released. The total volume of the reaction mixture was then made up to $35 \mathrm{ml}$ with $99.5 \%$ ethanol and the black solid product was separated by filtration. After washing with $50-150 \mathrm{ml}$ of $95 \%$ ethanol until a $\mathrm{pH}$ of about 5-6, it was finally air-dried. No special precaution was taken to prevent oxidation by air.

\subsection{Catalyst characterization}

The powder X-ray diffraction (XRD) patterns were recorded on non-oriented samples with a Scintag $\mathrm{X} 1$ diffractometer using copper $\mathrm{K}$ alpha radiation (wavelength $=0.154 \mathrm{~nm}$ ). Nitrogen adsorptiondesorption isotherms were obtained at $77 \mathrm{~K}$ on a Micromeritics ASAP 2000 apparatus. The data were analyzed with BET equation for surface area and BJH method for pore size distribution. Elemental composition was analyzed with a simultaneous ICP-AES allied analytical system (Jarrel-Ash, Model ICAP 9000).

Nickel K-edge extended X-ray absorption fine structure (EXAFS), soft X-ray absorption (XAS) and $\mathrm{X}$-ray photoelectron (XPS) spectroscopic studies were performed at the Synchrotron Radiation Research Center in Hsinchu, Taiwan. The storage ring was operated at about $1.5 \mathrm{GeV}$ with a ring current of about $200 \mathrm{~mA}$. EXAFS experiments were done in transmission mode with ionization detectors. The sample was pressed into pellet and sat within the sample holder of a home-made in-situ cell. For the in-situ reduction experiment, the sample was heated to the required temperature at a rate of $5^{\circ} \mathrm{C} / \mathrm{min}$ and maintained for about $1 \mathrm{~h}$. XAS experiments were done in sample current mode with electron yield detectors. The samples were evacuated under vacuum overnight before measurement was made at room temperature. XPS experiments were carried out with an incident X-ray energy of $375 \mathrm{eV}$. The peak areas of silicon (2p) and nickel (3p) were determined by standard peak fitting program. 


\subsection{Catalytic reaction}

Hydrogenation of benzene was carried out in a continuous flow micro-reactor system at atmospheric pressure. The sample was pretreated at various temperatures under a flowing $10 \%$ hydrogen/nitrogen gas mixture for $1 \mathrm{~h}$ before reaction. Reaction was started at $150^{\circ} \mathrm{C}$ by flowing pure hydrogen $(18 \mathrm{ml} / \mathrm{min})$ through a benzene reservoir maintained at temperature between $1-2^{\circ} \mathrm{C}$. The reaction product (mainly cyclohexane) was analyzed on-line by a Shimadzu GC-14B gas chromatograph equipped with a FID and capillary column (DB-1, $60 \mathrm{~m} \times 0.25 \mathrm{~mm}$ ). The reaction conditions are described in the result section.

\section{Results and discussion}

\subsection{Catalyst preparation}

In the preparation of a supported catalyst, maintaining the structural integrity of the support is of utmost importance since catalytic activity and product selectivity depend on it. However, sodium borohydride solution is strongly basic even with ethanol as the solvent, and this ethanolic solution is detrimental to the structure of pure MCM-41. For example, a blank test with $0.15 \mathrm{~g}$ of sodium borohydride in $4 \mathrm{ml}$ of ethanol causes complete dissolution of MCM-41 framework. The destruction of MCM-41 structure can be caused by the nucleophilic attack of framework silicon by ethoxide ion supply continuously via the slow reaction between sodium borohydride and ethanol (refer to Eqs. (2) and (3)). Therefore, the preparation method designed by Wang et al. [10] for the preparation of $\mathrm{Ni}-\mathrm{B} / \mathrm{SiO}_{2}$ is not suitable for MCM-41 since the first step involved immersing the support into borohydride solution prior to nickel chloride solution. In order to prepare Ni-B/MCM-41 catalyst successfully, we modified their method by reversing the order of reactant addition.

The presence of nickel chloride on MCM-41 surface before contacting with sodium borohydride can improve the structural integrity of MCM-41 in the catalyst significantly (Fig. 1). However, the amount of nickel chloride initially loaded on MCM-41 surface should not be too low. Apparently, the reduction of nickel cations by sodium borohydride now becomes

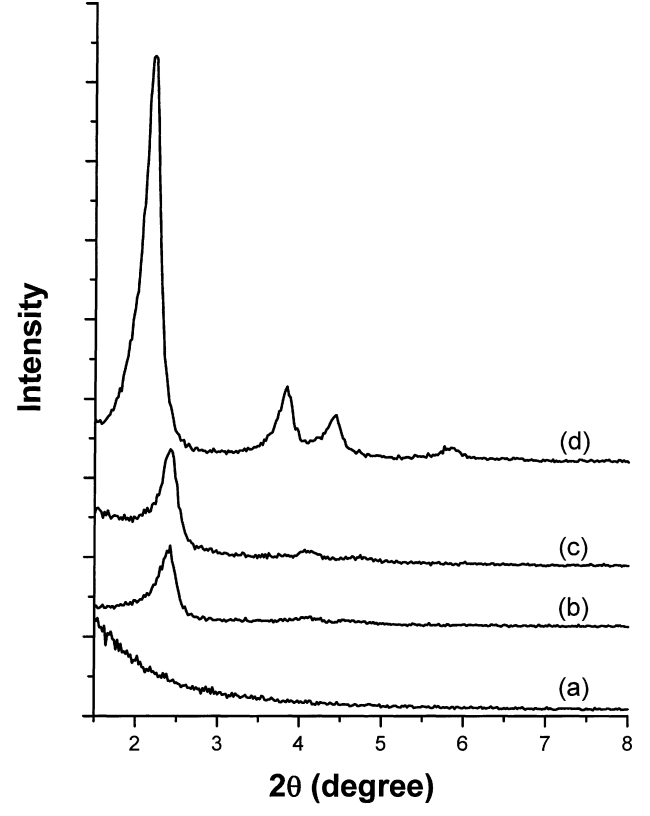

Fig. 1. XRD patterns of (a) S10; (b) M8; (c) M14; and (d) MCM-41.

dominant over the production of ethoxide ions. Hence, far less ethoxide ions are present in the reaction medium than the previous case without nickel cations. Moreover, reduction of nickel cations liberate protons, which lower the $\mathrm{pH}$ of the reaction medium (refer to Eq. (1)) [4]. Both these factors contribute to the stability of MCM-41. However, when the reduction was carried out in an aqueous medium where hydroxide ions are present, the MCM-41 structure is destroyed even with an initial nickel loading of $30 \mathrm{wt} . \%$.

Fig. 1 compares the XRD patterns of pristine MCM-41 and three different catalysts listed in Table 1. The intensity of the first diffraction peak of MCM-41 in the catalysts decreased drastically together with a slight shift to lower d-spacing. In addition, the higher order peaks also nearly disappeared. Therefore, the MCM-41 framework structure in the catalyst is more disordered under the influence of surface-adsorbed species. Not surprisingly, no diffraction peaks are observed for amorphous silica-supported catalyst.

Fig. 2 compares the nitrogen adsorption-desorption isotherms of MCM-41 and two typical Ni-B/MCM-41 catalysts. The pore size distribution of M8 (Fig. 2b) and also M6 is not much different from pristine 
Table 1

Physical characterization of $\mathrm{Ni}-\mathrm{B} / \mathrm{SiO}_{2}$ and $\mathrm{Ni}-\mathrm{B} / \mathrm{MCM}-41$ catalysts ${ }^{\mathrm{a}}$

\begin{tabular}{llllcrr}
\hline Sample & $\mathrm{Ni}_{\mathrm{I}}(\mathrm{wt} . \%)$ & $\mathrm{B} / \mathrm{Ni}$ & Composition & $\mathrm{Ni}_{\mathrm{F}}(\mathrm{wt} . \%)$ & $\mathrm{S} / \mathrm{A}\left(\mathrm{m}^{2} / \mathrm{g}\right)$ & $\mathrm{P} / \mathrm{V}(\mathrm{ml} / \mathrm{g})$ \\
\hline MCM-41 & - & - & - & - & 1051 & 0.93 \\
M6 & 30 & 0.7 & $\mathrm{Ni}_{75.3} \mathrm{~B}_{24.7}$ & 6.9 & 757 & 27.0 \\
M8 & 15 & 2.0 & $\mathrm{Ni}_{67.7} \mathrm{~B}_{32.3}$ & 10.6 & 739 & 0.60 \\
M14 & 10 & 6.1 & $\mathrm{Ni}_{62.1} \mathrm{~B}_{37.9}$ & 6.9 & 465 & 0.58 \\
S10 & 15 & 2.0 & $\mathrm{Ni}_{61.1} \mathrm{~B}_{38.9}$ & 7.5 & 394 & 0.37 \\
\hline
\end{tabular}

${ }^{a} \mathrm{M}$ and $\mathrm{S}$ denote MCM-41 and amorphous silica-supported catalysts, respectively. $\mathrm{Ni}_{\mathrm{I}}$ and $\mathrm{Ni}_{\mathrm{F}}$ represent the initial and final loading of nickel on the support, respectively. S/A, P/V and P/D denote the surface area, pore volume, and pore diameter of the sample, respectively.

MCM-41 judging from the steep rise in the inflexion region. The size of their hexagonal channels remained close to $27 \AA$. In addition, the much lower pore volumes of the catalysts compared to pristine MCM- 41 indicate the presence of surface species in the channel system. When the $\mathrm{B} / \mathrm{Ni}$ molar ratio was increased to 6, this $27 \AA$ pore structure collapsed and two new pore structures are formed (M14 in Fig. 2a). Now, the hysteresis at $P / P_{0}>0.4$ also becomes more prominent. Table 1 summarized the preparative conditions and characterization results of the catalysts.

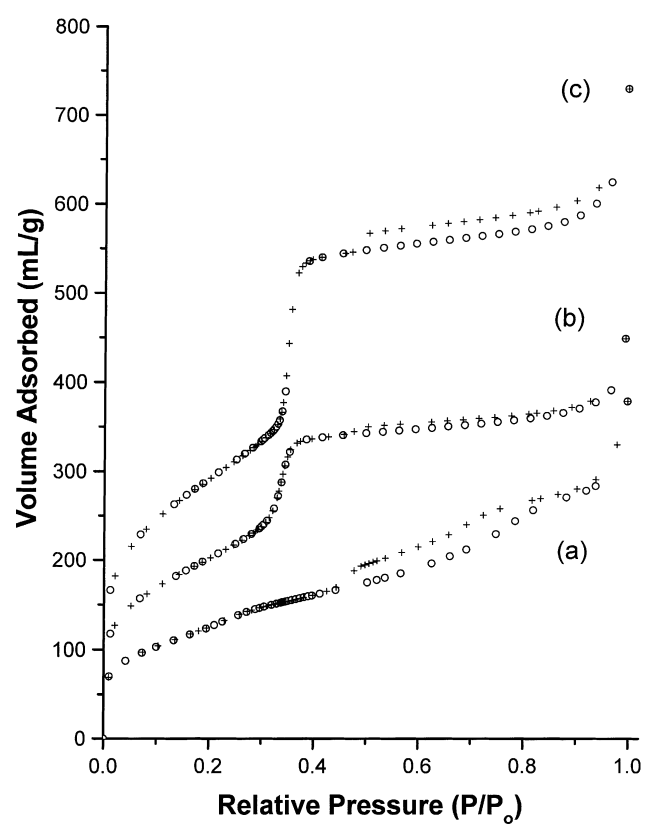

Fig. 2. Nitrogen adsorption-desorption isotherms of (a) M14; (b) M8; and (c) MCM-41. Top curve: desorption, bottom curve: adsorption.
Generally, the formation of Ni-B alloy is thought to involve two major processes, which contribute to the respective element of the alloy, namely: (I) reduction of nickel cation, and (II) decomposition of sodium borohydride [4]. Process II is initiated by nickel atoms formed in process I. In the confined environment of MCM-41 channels, it is possible that process II is suppressed due to deficiency in sodium borohydride that is not replenished as fast as in the case of homogenous reaction. Besides, the uni-dimensional channel system restricts inter-channel mixing of nickel and boron atoms formed from processes I and II. Therefore, it is reasonable to observe segregated nickel and boron phases, other than Ni-B alloy, on the surface of Ni-B/MCM-41 catalysts (see later).

One should also note that the formation of a segregated boron phase is inevitable even with amorphous support. In the aqueous synthesis reaction, for example, the formation of boric acid $\left(\mathrm{HBO}_{2}\right)$ on the support via a hydrolysis process (process III) is inevitable. In our case of non-aqueous synthesis method, we have found that a large fraction of the boron species formed is water-soluble. When sample S10 was subjected to additional flushing with $200 \mathrm{ml}$ of water during the filtration step, the amount of boron decreased by $73 \%$ as compared to $16 \%$ for nickel. The composition of the resulting surface species is now $\mathrm{Ni}_{82.6} \mathrm{~B}_{17.4}$. Therefore, not all the boron content listed in Table 1 is associated with nickel.

With reference to the reaction equations proposed for aqueous phase reaction, we suggest three corresponding equations for the interaction between nickel chloride and sodium borohydride in ethanolic solution:

$$
\begin{aligned}
& \mathrm{BH}_{4}^{-}+2 \mathrm{Ni}^{2+}+3 \mathrm{C}_{2} \mathrm{H}_{5} \mathrm{OH} \\
& \quad \rightarrow 2 \mathrm{Ni}+3 \mathrm{H}^{+}+2 \mathrm{H}_{2}+\mathrm{B}\left(\mathrm{OC}_{2} \mathrm{H}_{5}\right)_{3}
\end{aligned}
$$




$$
\begin{aligned}
& \mathrm{BH}_{4}{ }^{-}+\mathrm{C}_{2} \mathrm{H}_{5} \mathrm{OH} \rightarrow \mathrm{B}+\mathrm{C}_{2} \mathrm{H}_{5} \mathrm{O}^{-}+2.5 \mathrm{H}_{2} \\
& \mathrm{BH}_{4}{ }^{-}+4 \mathrm{C}_{2} \mathrm{H}_{5} \mathrm{OH} \\
& \quad \rightarrow \mathrm{B}\left(\mathrm{OC}_{2} \mathrm{H}_{5}\right)_{3}+\mathrm{C}_{2} \mathrm{H}_{5} \mathrm{O}^{-}+4 \mathrm{H}_{2}
\end{aligned}
$$

Judging from the equations proposed above, there are probably some triethylborate molecules (side product of boron) still adsorbed on the surface of the catalyst. This compound decomposes readily by water to form the water-soluble boron species.

\subsection{Nature of surface species}

The reaction of sodium borohydride with nickel chloride is a fast and vigorous process where the black or greyish black colored Ni-B alloy is formed immediately after the two reactants were mixed. However, we found that Ni-B/MCM-41 catalysts are susceptible to oxidation by air at room temperature since the black color of the as-synthesized sample change to grey usually within $1 \mathrm{~h}$ after it was separated from the solution. The color of the sample faded even further to light grey or dirty green after prolong storage in sample bottle. Conversely, the black color of $\mathrm{Ni}-\mathrm{B} / \mathrm{SiO}_{2}$ catalyst is not much changed even after a few months. The lower stability of Ni-B/MCM-41 catalyst towards oxidation than $\mathrm{Ni}-\mathrm{B} / \mathrm{SiO}_{2}$ suggests that its alloy particles are smaller, and oxygen adsorption on the surface affects a larger proportion of Ni-B interactions. This suggestion is reasonable considering that the channel system of MCM-41 will limit the growth of particle size.

\subsubsection{Characterization by X-ray diffraction technique}

Since the surface species in the as-synthesized catalysts are initially amorphous in nature, we then subjected the Ni-B/MCM-41 and $\mathrm{Ni}-\mathrm{B} / \mathrm{SiO}_{2}$ samples to a thermal treatment process (nitrogen, $450^{\circ} \mathrm{C}, 2 \mathrm{~h}$ ) in order to study the crystallization process of these species [10]. As shown in Fig. 3, the resultant species formed on MCM-41 and amorphous silica are different. Metallic nickel is formed on amorphous silica, which is consistent with literature finding that thermal treatment of alloy particles causes aggregation of nickel atoms and increased the surface content of boron oxide [11]. Nickel oxide is also present

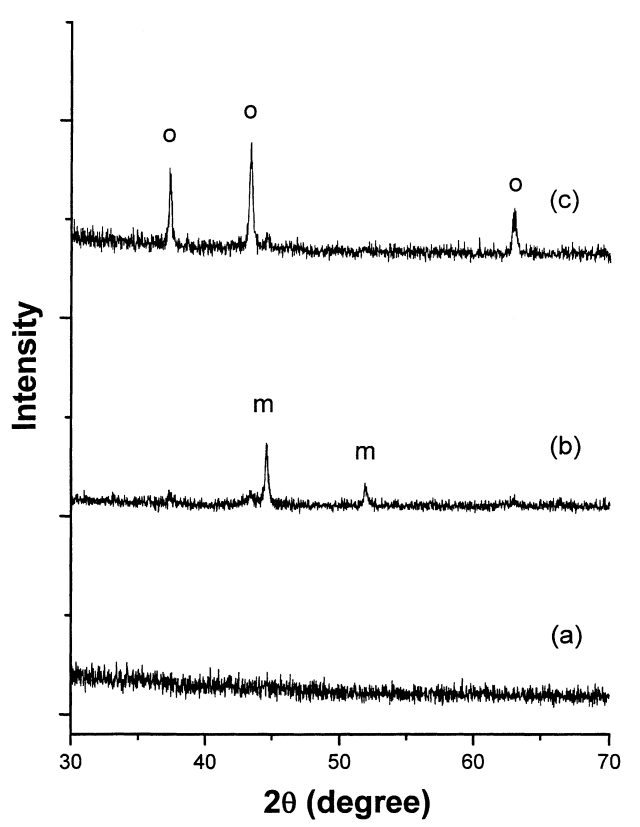

Fig. 3. XRD patterns of (a) M14; (b) S10; and (c) M8. o = nickel oxide, $\mathrm{m}=$ metallic nickel.

but the peak is much less intense. On the other hand, much more nickel oxide is present on MCM-41 (samples M8, and also M6). Thus, a significant amount of the nickel atoms on the as-synthesized Ni-B/MCM-41 catalyst is not involved in the formation of $\mathrm{Ni}-\mathrm{B}$ alloy but remained as a segregated nickel phase. This nickel phase was then oxidized to the oxide phase upon exposure to air. The Ni-B phase, if exist, should be in much smaller quantity than that on amorphous silica.

Moreover, XRD can only detect particles with size larger than about $50 \AA$, which exceeds the $27 \AA$ pore diameter of MCM-41 mesoporous channels [12]. Therefore, the nickel metal or oxide particles formed after thermal treatment of Ni-B/MCM-41 should be located on the external surfaces of MCM-41.

There are no large particles of nickel species observed in M14 after thermal treatment, even though its surface area is lower than M8. Possibly, the collapsed structure inhibits the mobility of surface species. However, the effect of boron content cannot be neglected. Li and Coville [13] have shown that boron suppresses the formation of large crystalline $\mathrm{Co}_{3} \mathrm{O}_{4}$ on $\mathrm{Co} / \mathrm{TiO}_{2}$ catalyst. 


\subsubsection{Characterization by X-ray absorption technique}

In order to see whether the nickel species have migrated from the channels to the external surfaces of MCM-41, we compared the XPS relative peak areas of nickel and silicon $(\mathrm{Ni} / \mathrm{Si})$ for various samples before and after thermal treatment at $450^{\circ} \mathrm{C}$. XPS is a surface sensitive technique. Using the standard equation $d=0.011 E^{3 / 2} / \rho$, the escape depth $(d)$ for the photoelectrons is estimated to be less than $9 \AA$ with nickel oxide as the reference material [14]. Here, $E$ is the energy of the photoelectrons in electron volt and $\rho$ is the density of the material under study in gram per cubic centemeter. If migration of nickel species occurs, then Ni/Si should increase following crystallization of nickel species on the external surface of MCM-41. We found that this ratio increased from 0.07 to 0.12 for M8, and from 0.22 to 0.34 for M6. However, the increase is not large indicating that the migration of nickel species is not serious. On the other hand, this ratio should decrease if no migration of nickel species occur. In fact, the ratio decreased from 0.47 to 0.27 for S10 after crystallization of surface species.

The surface species in the catalysts was further examined by XAS. In our experiments, the escape depth for Auger electrons is estimated to be about $40 \AA$ with nickel oxide as the reference material. Therefore, it is possible to observe the surface species in the $27 \AA$ mesoporous channels using this technique. We must emphasize that the shielding effect of the near $20 \AA$ silica channel wall should not be too great since the density of silica is much lower than the alloy.

The Ni $2 \mathrm{p}_{3 / 2}$ and $2 \mathrm{p}_{1 / 2}$ absorption spectra corresponding to the $\mathrm{Ni} 2 \mathrm{p} \rightarrow 3 \mathrm{~d}$ transitions for $\mathrm{Ni}-\mathrm{B} / \mathrm{MCM}-41$ and $\mathrm{Ni}-\mathrm{B} / \mathrm{SiO}_{2}$ catalysts are shown in Fig. 4. Comparing the spectra of the Ni-B/MCM-41 and $\mathrm{Ni}-\mathrm{B} / \mathrm{SiO}_{2}$ catalysts shows that their surface nickel atoms have similar oxidation states. The main $\mathrm{Ni} 2 \mathrm{p}_{3 / 2}$ peak has similar absorption energy to metallic nickel (standard value $=851.5 \mathrm{eV}$ ), although a slight shift (maximum $0.26 \mathrm{eV}$ ) towards higher energy is sometimes observed. The shoulder at higher energy corresponds to nickel oxide, and it is more resolved in the as-synthesized Ni-B/MCM-41 catalyst. Here, the proportion of metallic nickel observed in the catalysts is higher than nickel oxide. Possibly, some of the

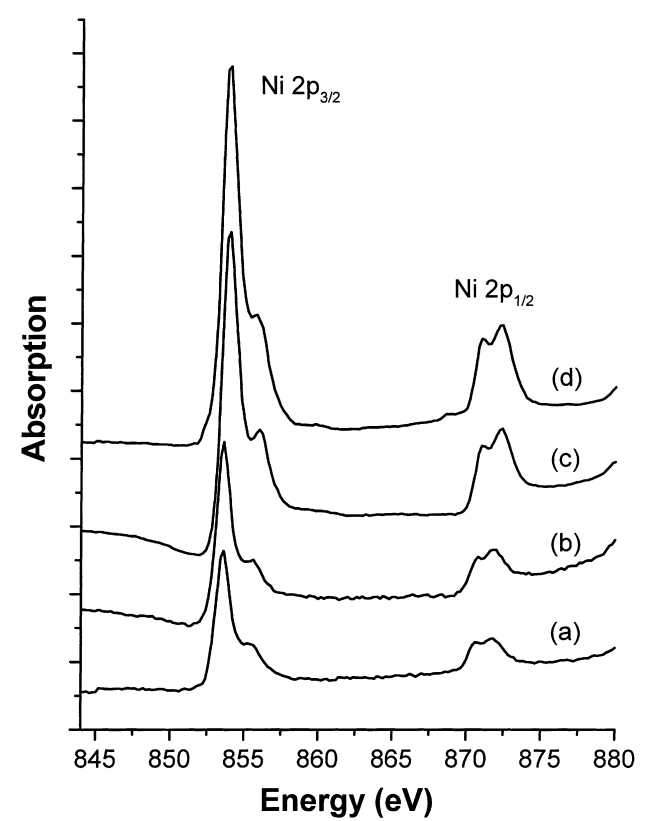

Fig. 4. Soft X-ray absorption spectra of (a) S10, treated at $450^{\circ} \mathrm{C}$ under nitrogen; (b) M8, treated at $450^{\circ} \mathrm{C}$ under nitrogen; (c) M8, as-synthesized; and (d) S10, as-synthesized.

oxygen adsorbed on nickel in these catalysts is easily removed by evacuation. The peak maximum shifts slightly to lower energy after thermal treatment for all samples indicating a certain degree of reduction of the catalysts.

We have also analyzed the B 1s absorption spectra corresponding to the $1 s \rightarrow 2 p$ transition. The escape depth is estimated to be less than $10 \AA$. Unfortunately, the signal to noise ratio is very low due to the low surface concentration of boron in these samples. Meaningful quantitative information cannot be extracted from the spectrum.

EXAFS study can provide us detailed information on the nature of the surface species on the catalysts. The nickel $\mathrm{K}$-edge radial distribution functions (RDFs) of both $\mathrm{Ni}-\mathrm{B} / \mathrm{MCM}-41$ and $\mathrm{Ni}-\mathrm{B} / \mathrm{SiO}_{2}$ catalysts are compared in Fig. 5. The RDFs usually consist of three main peaks, which were assigned at increasing bond distance to $\mathrm{Ni}-\mathrm{O}, \mathrm{Ni}-\mathrm{Ni}$ (metal) and $\mathrm{Ni}-\mathrm{Ni}$ (oxide) neighbors, respectively. These assignments were made according to the published results of Sano et al. [15]. Furthermore, the peak height 


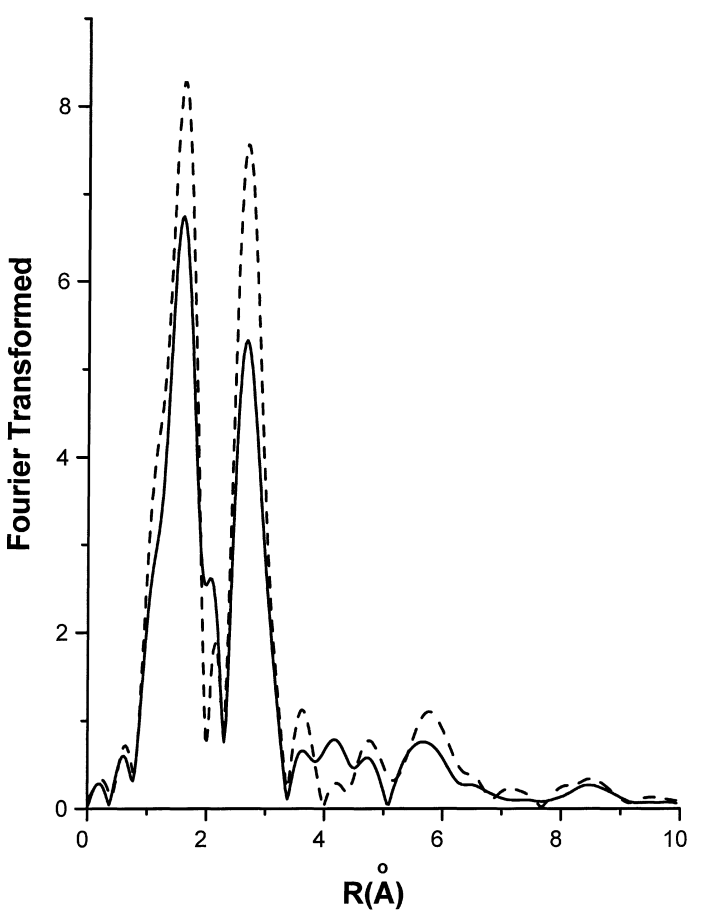

Fig. 5. A comparison of the EXAFS RDFs of M8 (dash line) and S10 (solid line).

corresponds to the average coordination number of the respective neighbor around the central nickel atom.

All the Ni-B/MCM-41 catalysts showed similar RDFs. It is important to note that the Ni-Ni (metal) peak which appeared at a bond distance of about $2 \AA$ resembles that of Ni-B alloy in $\mathrm{Ni}-\mathrm{B} / \mathrm{SiO}_{2}$ catalyst reported by Wang et al. [16]. This peak usually has low intensity. The presence of this peak suggests that the oxidation of alloy particles is not complete and may be limited to the external surface. We have also found that the intensity of this peak is higher in $\mathrm{Ni}-\mathrm{B} / \mathrm{SiO}_{2}$ than Ni-B/MCM-41 due to its larger particle size. As a result, a larger fraction of Ni-B interactions is protected from oxidation by staying in the bulk. The lower intensity of $\mathrm{Ni}-\mathrm{O}$ and $\mathrm{Ni}-\mathrm{Ni}$ (oxide) peaks in $\mathrm{Ni}-\mathrm{B} / \mathrm{SiO}_{2}$ also supports our previous observation that $\mathrm{Ni}-\mathrm{B} / \mathrm{SiO}_{2}$ is less susceptible to oxidation than Ni-B/MCM-41. One should note that hydrogen chemisorption is not suitable for studying the particle size (dispersion) of Ni-B alloy in this case since it cannot differentiate between nickel atoms in nickel

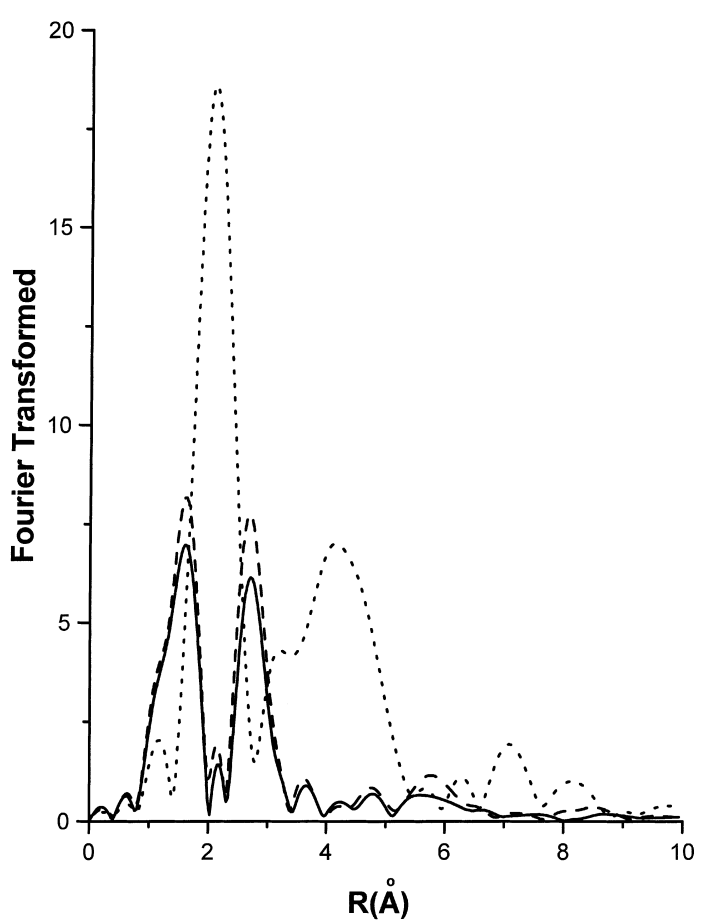

Fig. 6. EXAFS RDFs of M6 reduced at different temperatures: as-synthesized (dash line), reduced at $150^{\circ} \mathrm{C}$ (solid line), and reduced at $300^{\circ} \mathrm{C}$ (dotted line).

oxide and nickel boride phases. Therefore, EXAFS is a more appropriate technique here.

We have also carried out an in-situ EXAFS study on the reduction of $\mathrm{Ni}-\mathrm{B} / \mathrm{MCM}-41$ catalyst with pure hydrogen at different temperatures (Fig. 6). When the Ni-B/MCM-41 sample was reduced at $300^{\circ} \mathrm{C}$, the $\mathrm{Ni}-\mathrm{Ni}$ (metal) peak gains intensity significantly due to the sintering of nickel metal particles. These sintered nickel particles are resistant to oxidation by air. XRD analysis of this sample also revealed a small diffraction peak at $d=2.03 \AA$ due to metallic nickel. The same peak is also observed in $\mathrm{Ni}-\mathrm{B} / \mathrm{SiO}_{2}$ catalyst reduced at $300^{\circ} \mathrm{C}$. Clearly, the interactions between nickel and boron in the alloy were destroyed by reduction at this temperature. We are still not clear why the crystallization temperature of nickel is much lower under hydrogen atmosphere than nitrogen atmosphere. Possibly, the boron in the alloy or the protective surface oxide coating is loss in a hydrogen environment. A similar phenomenon has been observed for other alloy systems such as $\mathrm{Fe}-\mathrm{B}$ [1]. At $430^{\circ} \mathrm{C}$, this $\mathrm{Ni}-\mathrm{Ni}$ 
(metal) peak becomes as intense as the corresponding peak from nickel foil (figure not shown).

\subsection{Catalysis}

In order to understand further the surface state of the as-synthesized Ni-B/MCM-41 and $\mathrm{Ni}-\mathrm{B} / \mathrm{SiO}_{2}$ catalysts, we employed a catalytic benzene hydrogenation reaction as the reaction probe. All the reactions were carried out at $150^{\circ} \mathrm{C}$ but the catalysts were pre-reduced at different temperatures before the reaction. From Fig. 7, it is clear that Ni-B/MCM-41 catalyst is less active than $\mathrm{Ni}-\mathrm{B} / \mathrm{SiO}_{2}$ catalysts pretreated at the same $\left(220^{\circ} \mathrm{C}\right)$ or lower $\left(150^{\circ} \mathrm{C}\right)$ temperatures. Apparently, $\mathrm{Ni}-\mathrm{B} / \mathrm{SiO}_{2}$ catalyst has much more active sites for the reaction than $\mathrm{Ni}-\mathrm{B} / \mathrm{MCM}-41$, which means that more surface nickel sites are being reduced at the above temperatures. We have shown previously that a segregated nickel oxide phase is present on Ni-B/MCM-41 catalysts. Possibly, the reducibility of nickel atoms in nickel oxide phase is relatively lower than those in

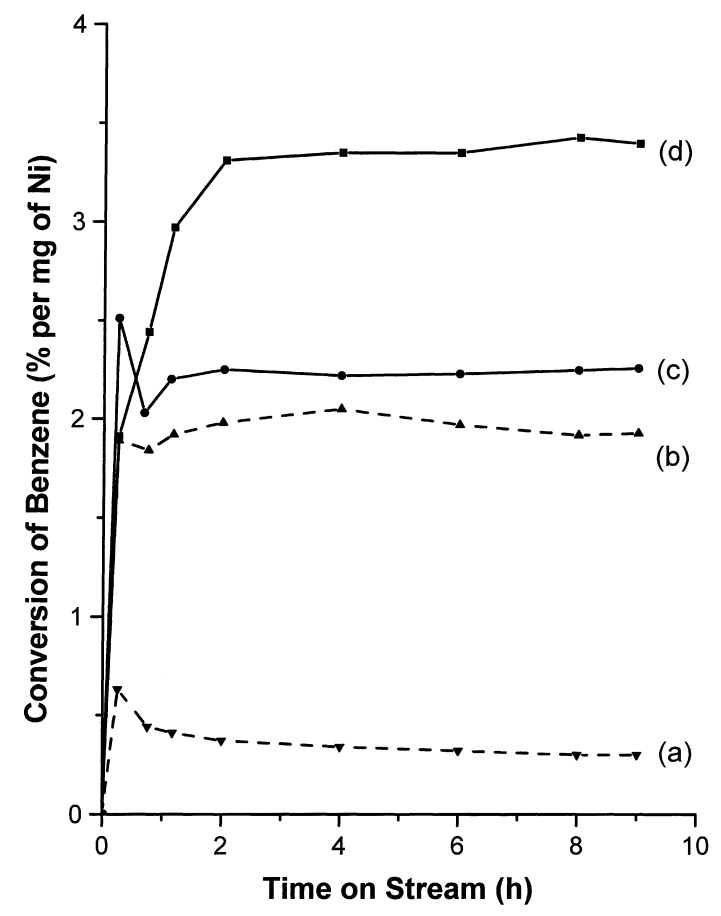

Fig. 7. Benzene conversion at $150^{\circ} \mathrm{C}$ vs. reaction time on stream: (a) M6, pretreated at $220^{\circ} \mathrm{C}$; (b) M6, pretreated at $300^{\circ} \mathrm{C}$; (c) $\mathrm{S} 10$, pretreated at $150^{\circ} \mathrm{C}$; and (d) $\mathrm{S} 10$, pretreated at $220^{\circ} \mathrm{C}$. nickel boride alloy phase. This phenomenon can be related to the electron donating and ensemble effect of boron, which will make the nickel-oxygen interactions weaker [17].

The activity of a completely reduced Ni-B/MCM-41 catalyst (at $300^{\circ} \mathrm{C}$ ) is still lower than the $\mathrm{Ni}-\mathrm{B} / \mathrm{SiO}_{2}$ catalyst pretreated at lower temperatures. At this stage, sintering of nickel atoms occurred leading to a decrease in the number of active sites responsible for benzene hydrogenation reaction. In fact, sintering process occurred at $300^{\circ} \mathrm{C}$ on both catalysts as shown by the in-situ EXAFS and XRD analysis. Undoubtedly, we have reached the Huttig temperature of nickel $\left(\approx 302^{\circ} \mathrm{C}\right)$ where migration of nickel atoms over the surface of nickel starts [18]. Therefore, it is reasonable to conclude that the silica surface of MCM-41 does not has the advantage of stabilizing the nickel species.

\section{Conclusion}

The high surface area and porosity of MCM-41 channel system is particularly useful for the dispersion of active materials during catalyst preparation. In Ni-B/MCM-41 catalysts, the alloy phase should be better dispersed than $\mathrm{Ni}-\mathrm{B} / \mathrm{SiO}_{2}$ since the particle size is smaller. However, the silica framework of MCM-41 is not stable in basic medium and catalyst preparation involving high $\mathrm{pH}$ will tend to cause structural collapse. We have shown that this shortcoming of MCM-41 can be overcome by varying the preparative conditions, such as the solvent of the reaction medium. Another concern of using MCM-41 as catalyst support is its poor stabilization effect on the supported phase. With reference to amorphous silica, we did not see any positive effect of MCM-41 channel system on slowing down nickel particle sintering. However, if anchoring sites (e.g. cationic sites) were present on MCM-41, then the resistance to metal particle sintering could be improved. Further studies with aluminosilicate MCM-41 as the catalyst support should be very interesting. Of course, for the Ni-B/MCM-41 catalysts to have potential industrial application, the homogeneity of the surface species on MCM-41 has to be improved. The presence of a large amount of nickel oxide on MCM-41 lowers the catalytic activity of $\mathrm{Ni}-\mathrm{B} / \mathrm{MCM}-41$ in benzene hydrogenation reaction. 
In conclusion, we found that the channel system of MCM-41 discourages free and homogeneous interaction between reacting species in the synthesis medium. This effect leads to the formation of different surface species in $\mathrm{Ni}-\mathrm{B} / \mathrm{SiO}_{2}$ and $\mathrm{Ni}-\mathrm{B} / \mathrm{MCM}-41$, i.e. amorphous Ni-B alloy in the former and mainly nickel oxide in the latter.

\section{Acknowledgements}

This research was supported by the China Petroleum Co. and the National Science Council of Taiwan (NSC88-2811-M002-0019).

\section{References}

[1] A. Molnar, G.V. Smith, M. Bartók, Adv. Catal. 36 (1989) 329. [2] W.L. Johnson, Prog. Mater. Sci. 30 (1986) 81.

[3] G.V. Smith, W.E. Brower, M.S. Matyjaszczyk, T. Pettit, in: Proceedings of the 7th International Congress Catalysis, Tokyo, 1980, p. 355.

[4] Y. Chen, Catal. Today 44 (1998) 3.
[5] J. Deng, X. Zhang, E. Min, Appl. Catal. 37 (1988) 339.

[6] W.-J. Wang, M.-H. Qiao, H. Li, J.-F. Deng, Appl. Catal. 166 (1998) L243.

[7] J.S. Beck, J.C. Vartuli, W.J. Roth, M.E. Leonowicz, C.T. Kresge, K.D. Schmitt, C.T.-W. Chu, D.H. Olson, E.W. Sheppard, S.B. McCullen, J.B. Higgins, J.L. Schlenker, J. Am. Chem. Soc. 114 (1992) 10834.

[8] M.C. Schoenmaker-Stolk, J.W. Verwijs, J.A. Don, J.J.F. Scholten, Appl. Catal. 29 (1987) 73.

[9] H.-P. Lin, S. Cheng, C.-Y. Mou, Microporous Mater. 10 (1997) 111.

[10] W.-J. Wang, M.-H. Qiao, J. Yang, S.-H. Xie, J.-F. Deng, Appl. Catal. 163 (1997) 101.

[11] H. Yamashita, M. Yoshikawa, T. Funabiki, S. Yoshida, J. Chem. Soc., Faraday Trans. 182 (1986) 1771.

[12] A.N. Desikan, L. Huang, S.T. Oyama, J. Phys. Chem. 95 (1991) 10050.

[13] J. Li, N.J. Coville, Appl. Catal. 181 (1999) 201.

[14] W.T. Elam, J.P. Kirkland, R.A. Neiser, P.D. Wolf, Phys. Rev. B 38 (1988) 26.

[15] M. Sano, T. Maruo, H. Yamatera, M. Suzuki, Y. Saito, J. Am. Chem. Soc. 109 (1987) 52.

[16] W.-J. Wang, M.-H. Qiao, H.-X. Li, W.-L. Dai, J.-F. Deng, Appl. Catal. 168 (1998) 151.

[17] S. Yoshida, H. Yamashita, T. Funabiki, T. Yonezawa, J. Chem. Soc., Faraday Trans. 180 (1984) 1435.

[18] M.C. Schoenmaker-Stolk, J.W. Verwijs, J.J.F. Scholten, Appl. Catal. 29 (1987) 91 